\title{
Postprandial hypotension as a risk marker for asymptomatic lacunar infarction.
}

\section{$\operatorname{AUTHOR}(\mathrm{S}):$}

Tabara, Yasuharu; Okada, Yoko; Uetani, Eri; Nagai, Tokihisa; Igase, Michiya; Kido, Tomoko; Ochi, Namiko; ... Takita, Rie; Kohara, Katsuhiko; Miki, Tetsuro

\section{CITATION:}

Tabara, Yasuharu ... [et al]. Postprandial hypotension as a risk marker for asymptomatic lacunar infarction.. Journal of hypertension 2014, 32(5): 1084-1090

\section{ISSUE DATE:}

2014-05

URL:

http://hdl.handle.net/2433/199874

\section{RIGHT:}

This is a non-final version of an article published in final form in [Postprandial hypotension as a risk marker for asymptomatic lacunar infarction. Tabara Yasuharu, Okada Yoko, Uetani Eri, Nagai Tokihisa, Igase Michiya, Kido Tomoko, Ochi Namiko, Ohara Maya b, Takita Rie, Kohara Katsuhiko, Miki Tetsuro. Journal of Hypertension. 32(5):1084. 1090, May 2014. DOI: 10.1097/HJH.0000000000000150.]; この論文は出版社版でありません。引用の際には出版社版を ご確認ご利用ください。; This is not the published version. Please cite only the published version. 
Title

Short title

Authors

Affiliation

Correspondence

Word count

Keywords
Disclosure

Postprandial hypotension as a risk marker for asymptomatic lacunar infarction: The J-SHIPP study

Postprandial hypotension and lacunar infarction

Yasuharu Tabara $\mathrm{PhD}^{1)}$ 2), Yoko Okada $\mathrm{MD}^{2)}$, Eri Uetani $\mathrm{MD}, \mathrm{PhD}^{2)}$, Tokihisa Nagai $\mathrm{MD}, \mathrm{PhD}^{2)}$, Michiya Igase $\mathrm{MD}, \mathrm{PhD}^{2)}$, Tomoko Kido $\mathrm{MD}, \mathrm{PhD}^{2)}$, Namiko Ochi MD, $\mathrm{PhD}^{2)}$, Maya Ohara $\mathrm{MD}^{2)}$, Rie Takita $\mathrm{MD}, \mathrm{PhD}^{2)}$, Katsuhiko Kohara $\mathrm{MD}, \mathrm{PhD}^{2)}$, Tetsuro Miki $\mathrm{MD}, \mathrm{PhD}^{2 \text { ) }}$

1. Center for Genomic Medicine, Kyoto University Graduate School of Medicine, Kyoto, Japan

2. Department of Geriatric Medicine, Ehime University Graduate School of Medicine

Yasuharu Tabara, PhD

Center for Genomic Medicine,

Kyoto University Graduate School of Medicine

Shogoinkawaramachi, Sakyo-ku, Kyoto 606-8507, Japan

Tel: +81-75-366-7407, Fax: +81-75-751-4167

e-mail: tabara@genome.med.kyoto-u.ac.jp

Sauce of Founding This study was supported by a Grant-in-Aid for Scientific Research from the Ministry of Education, Culture, Sports, Science and Technology of Japan; a Science and Technology Incubation Program in Advanced Regions from the Japan Science and Technology Agency; a Grant-in-Aid for Scientific Research from the Japan Arteriosclerosis Prevention Fund; a research grant from the Takeda Science Foundation; and a Research Promotion Award of Ehime University.

body 3,940 words (abstract to reference)

abstract 250 words

4 tables, 2 figures, 30 references

The authors have no disclosures to declare.

Postprandial hypotension, lacunar infarction, periventricular hyperintensity, microbleeds 


\section{ABSTRACT}

Objective: Increasing blood pressure (BP) variability is reported to be a cardiovascular risk factor. However, the clinical implications of postprandial hypotension, a commonly observed BP variability in elderly persons, are poorly understood. Here, we investigated possible associations between postprandial BP decline and asymptomatic cerebral damage in community residents.

Methods: Study subjects consisted of 1,308 general community residents ( $65 \pm 9$ years old). Postprandial BP change was calculated from systolic BP measured just before and 30 min after lunch. Postprandial hypotension was defined as a decline in systolic BP of more than 20 mmHg. The presence of asymptomatic cerebrovascular damage was evaluated by brain magnetic resonance imaging.

Results: Prevalence of lacunar infarction was significantly higher in subjects with postprandial hypotension ( $\mathrm{p}=0.004)$. A postprandial decline in systolic BP was linearly increased with the number of lacunar lesions (none, $n=1,200,-3.4 \pm 11.3 \mathrm{mmHg} ; 1$ lesion, $\mathrm{n}=82,-5.2 \pm 11.8 ; 2$ lesions, $\mathrm{n}=18,-6.9 \pm 11.5 ; 3$ lesions, $\mathrm{n}=7,-13.4 \pm 11.3 ; 4$ lesions, $\mathrm{n}=1,-27$; $\mathrm{p}=0.012)$. Although subjects with postprandial hypotension were older $(\mathrm{p}<0.001)$ and had higher preprandial BP $(\mathrm{p}<0.001)$ and faster pulse wave velocity $(\mathrm{p}=0.001)$, multivariate analysis adjusted for these covariates indicated that postprandial BP decline was an independent determinant for the number of lacunar infarctions $(\mathrm{p}=0.004)$. No significant associations were observed with grade of periventricular hyperintensity or frequency of microbleeds. These relationships were also found in an analysis based on central BP, whereas no superiority was seen in the analysis based on central BP.

Conclusions: Postprandial BP decline is an overlooked risk marker for asymptomatic lacunar infarction in community residents. 


\section{INTRODUCTION}

Blood pressure (BP) variability is reportedly associated with cardiovascular mortality and morbidity, particularly in frail elderly persons. Orthostatic hypotension is a well-investigated phenomenon that might be associated with cardiovascular and cerebrovascular morbidity [13] and mortality [4, 5]. A second BP variability that is frequently observed in elderly persons is a meal-related BP decline, termed postprandial hypotension (PHYPO), with a reported prevalence in institutionalized elderly of approximately 25\%-38\% [6-7]. Although few clinical studies of PHYPO have appeared, longitudinal studies with low-level-care residents clearly show the epidemiological significance of PHYPO as a risk marker of incident stroke and coronary events [6], as well as total mortality [6, 8]. We previously reported a crosssectional association between PHYPO and asymptomatic cerebrovascular damage, including lacunar infarction and periventricular hyperintensities (PVH), in patients hospitalized for essential hypertension [9]. These findings and the potential high frequency of PHYPO emphasize the importance of a comprehensive understanding of its clinical and epidemiological significance [10]. Sample size in these previous studies was small, however, and the subjects were frail elderly or inpatients with essential hypertension.

Here, to better understand the clinical and epidemiological significance of postprandial BP decline, we investigated the association between PHYPO and asymptomatic cerebrovascular damage in a community-dwelling elderly cohort. Given recent clinical studies suggesting the superiority of central aortic systolic blood pressure (cSBP) in predicting future cardiovascular event risks [11], we also measured postprandial cSBP change and investigated possible associations with cerebrovascular diseases. 


\section{SUBJECTS AND METHODS}

\section{Study subjects}

The study subjects were 1,308 apparently healthy middle-aged to elderly persons who were consecutive participants in the medical check-up program at Ehime University Hospital Antiaging Center from February 2006 to August 2012. This medical check-up is provided to general residents of Ehime Prefecture, Japan, and is specifically designed to evaluate agingrelated disorders, including atherosclerosis, cardiovascular disease, physical function and mild cognitive impairment [12-15]. Recruitment was performed via mass communications such as internet homepages, periodical newspapers in the local community, and commercial newspapers. Among 1,678 total participants, 1,308 persons who underwent brain magnetic resonance imaging (MRI) $(n=1,466)$ and postprandial BP measurements $(n=1,387)$; completed all clinical measurements $(n=1,380)$; and were free from any history of symptomatic coronary heart disease, including congestive heart failure $(n=1,322)$, hemorrhage stroke, and embolic cerebral infarction $(n=1,308)$ were included in this analysis. Brain MRI and postprandial BP measurements were performed at the request of the individual subject. All participants were physically independent and completed all clinical measurements. The present study was part of a series of studies which was approved by the ethics committee of Ehime University Graduate School of Medicine.

\section{BP measurements}

Brachial BP and radial arterial waveform were measured simultaneously (HEM-9000AI: Omron Healthcare, Kyoto, Japan) after 5 min rest in the sitting position. Briefly, brachial BP was measured on the right upper arm using a cuff-oscillometric device, and the radial arterial 
waveform was simultaneously obtained from the left wrist using a multi-element tonometric sensor. Radial augmentation index was calculated from the waveform as the ratio of the height of the late systolic peak to that of the first peak [16]. Absolute pressure of the late systolic peak was obtained by calibrating the radial waveform with brachial systolic BP (SBP) and considered to be the cSBP. The validity of estimating cSBP has been demonstrated by invasive simultaneous measurement of ascending aorta and radial artery pressure [17-19].

\section{Evaluation of postprandial BP change}

Postprandial changes in BP were calculated from two readings in a single day, one obtained just before lunch and the second at 30 min after the lunch. Subjects were moved to an examination room afrer finishing the lunch, and postprandial BP was measured after at least 15 min lecture on dietetics. In both measurements, a single reading was obtained.

Postprandial BP change was calculated by subtracting preprandial SBP from postprandial SBP. Postprandial hypotension (PHYPO) was defined analgously to orthostatic hypotension as a posptprandial decrease in SBP $(\triangle \mathrm{SBP})$ of more than $20 \mathrm{mmHg}$. Subjects were classified into three groups according to $\Delta \mathrm{SBP}$, namely PHYPO: $\Delta \mathrm{SBP} \leq-20 \mathrm{mmHg}$, control $(-20<\Delta \mathrm{SBP}$ $\leq 10 \mathrm{mmHg}$ ), and postprandial hypertension (PHT: $\triangle \mathrm{SBP}>10 \mathrm{mmHg}$ ). Subjects were also classified according to the postprandial changes in cSBP $(\Delta \mathrm{cSBP})$, namely $\mathrm{PHYPO}(\Delta \mathrm{cSBP} \leq-$ $25 \mathrm{mmHg})$, control (-25 $<\Delta \mathrm{cSBP} \leq 5 \mathrm{mmHg})$, and PHT ( $\Delta \mathrm{cSBP}>5 \mathrm{mmHg}$ ). Lunch consisted of a traditional Japanese meal (600 kcal) for all subjects.

\section{MRI and assessment of silent cerebral damage}

The presence of asymptomatic cerebrovascular damage, including lacunar infarctions, PVH, 
and microbleeds was evaluated by brain MRI with a 3-tesla scanner (Signa Excite 3.0T; GE Healthcare., Milwaukee, WI). MRI was performed within one month before the clinical

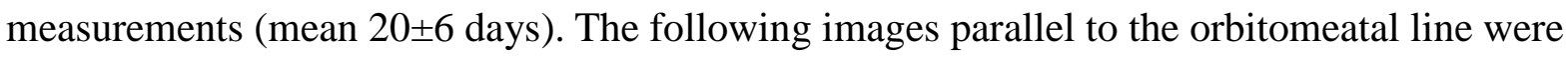
obtained: T1-weighted axial images (TR 2000.0 msec, TE 16.0 msec, thickness 6.0 mm, gap 1.0 mm, matrix 288x224), T2-weighted axial images (TR $4800.0 \mathrm{msec}$, TE $92.0 \mathrm{msec}$, thickness $6.0 \mathrm{~mm}$, gap $1.0 \mathrm{~mm}$, matrix 512x256), fluid-attenuated inversion recovery (FLAIR) images (TR $11000.0 \mathrm{msec}$, TE $140.0 \mathrm{msec}$, thickness $6.0 \mathrm{~mm}$, gap $1.0 \mathrm{~mm}$, matrix 288x224), and gradient echo (T2*-weighted) images (TR 600.0 msec, TE 7.2 msec, thickness $6.0 \mathrm{~mm}$, gap $1.0 \mathrm{~mm}$, matrix 320x192).

Lacunar infarction was defined as areas of low signal intensity (3- to 15-mm diameter) on T1-weighted images and of high intensity on T2-weighted and FLAIR images. Microbleeds were defined as small (2- to 5-mm diameter) hypointense lesions on $\mathrm{T} 2 *$-weighted images. Hypointense lesions within the subarachnoid space and areas of symmetric hypointensity in the globus pallidus on $\mathrm{T} 2 *$-weighted images were considered likely to represent adjacent pial blood vessels and calcifications, respectively, and were therefore excluded. PVH were defined as white matter hyperintensities depicted on T2-weighted and FLAIR images in contact with the ventricular wall. PVH was further classified into five grades, namely grade 0 , absent or only a "rim”; grade 1, limited lesion-like “caps”; grade 2, irregular "halo”; grade 3, irregular margins and extension into the deep white matter; and grade 4, extension into the deep white matter and subcortical portion. Imaging analysis was performed by neurologists without clinical information on the subject. Images were analyzed using the OsiriX software [20]. 


\section{Evaluation of arterial properties}

We measured carotid intima-media thickness (IMT) and brachial-to-ankle pulse wave velocity (baPWV) as indices of arteriosclerosis. To measure IMT, ultrasonography of the common carotid artery was performed using an SSD-3500SV or $\alpha 10$ ultrasonograph (Aloka Co, Ltd., Tokyo, Japan) with a 7.5-MHz probe. After 5 min rest in the supine position, optical visualization of the bilateral carotid arteries was obtained with the subject's head tilted slightly upward in the mild-line position. IMT of the far wall was measured from B-mode images using onboard computerized software, which simultaneously measured IMT at three points at 1-cm intervals. Nine IMTs of the far wall were measured at 1-cm intervals proximal to the bulb from the anterior, lateral and posterior approaches. Mean IMT calculated from the nine readings was used in the analysis. No measurements were taken at the level of a discrete plaque.

baPWV was measured in the supine position after at least 5 min rest. Cuffs were applied to both brachia and ankles, and all blood pressures (BPs) were measured simultaneously by the cuff-oscillometric method (BP-203RPEII (form PWV/ABI), Omron Healthcare). The pulse volume waveforms were also recorded simultaneously using a plethymographic sensor connected to the cuffs. baPWV was calculated from the time interval between the wave fronts of the brachial and ankle waveforms, and the path length from the brachia to ankle. A brief explanation of this device as well as the validity and reproducibility of its measurements have been provided elsewhere [21].

\section{Statistical analysis}

Mean values of continious variables were compared with ANOVA, while frequency 
differences were assessed using the chi-squared test. Multiple poisson regression analysis was used to identify factors independently associated with the number of lacunar lesion. All statistical analyses were conducted using commercially available statistical software (JMP ver 9.0.2; SAS Institute Inc., Cary, NC) or R software (http://www.r-project.org/) with p-values less than 0.05 considered statistically significant.

\section{RESULTS}

Clinical characteristics of the study subjects are summarized in Table 1 . Mean $\Delta$ SBP was 3.7 $\pm 11.4 \mathrm{mmHg}$ and was strongly correlated with that of $\mathrm{DBP}$ (mean $\Delta \mathrm{DBP}=-5.7 \pm 6.6$ mmHg; $\mathrm{r}=0.670, \mathrm{p}<0.001$ ). $\Delta \mathrm{SBP}$ was also strongly correlated with $\Delta \mathrm{cSBP}$ (Figure 1 ) $(\mathrm{r}=0.923, \mathrm{p}<0.001)$, and the coefficient of the regression line was 1.0. No significant class effects of antihypertensive drugs were seen for $\Delta$ SBP (calcium-channel blockers, $-2.4 \pm 11.7$ vs. other drugs $-4.2 \pm 13.7, \mathrm{p}=0.191$; angiotensin II receptor blockers, $-3.4 \pm 12.9$ vs. $-2.7 \pm 12.0$, $p=0.592$; angiotensin-converting enzyme inhibitors, $-6.7 \pm 13.6$ vs. $-2.9 \pm 12.4, p=0.229$; diuretics, $2.2 \pm 11.2$ vs. $-3.3 \pm 12.5, \mathrm{p}=0.053$; alpha-blockers, $-0.5 \pm 13.5$ vs. $-3.1 \pm 12.4, \mathrm{p}=0.487$; beta-blockers, $-4.8 \pm 15.0$ vs. $-2.8 \pm 12.1, \mathrm{p}=0.322)$.

PHYPO subjects defined by $\triangle \mathrm{SBP}$ had a greater number of lacunar infarctions (Table 2). On classification of subjects into the four subgroups of PHYPO-2 $\Delta \mathrm{SBP} \leq-20 \mathrm{mmHg}$, PHYPO-1 -20< $<\mathrm{SBP} \leq-10 \mathrm{mmHg}$, control $-10<\Delta \mathrm{SBP} \leq 10 \mathrm{mmHg}$, and PHT $\Delta \mathrm{SBP}>10 \mathrm{mmHg}$, lacunar infarction was more frequent in the PHYPO-2 (12.7\%) and PHYPO-1 (11.4\%) groups than in the control (8.1\%) and PHT (7.2\%) subjects $(\mathrm{p}=0.007)$. We tentatively defined a cutoff point of $\Delta \mathrm{cSBP}$ for postprandial hypotension and hypertension as -25 and $5 \mathrm{mmHg}$ respectively, because the intercept of the regression line between $\Delta$ SBP and $\Delta \mathrm{cSBP}$ was -4.3 
mmHg (Figure 1). In this analysis using $\Delta \mathrm{cSBP}$ as an index of postprandial BP change, postprandial hypotension was also significantly associated with a greater number of lacunar infarctions (Table 2).

Differences in clinical parameters by the number of lacunar lesions are summarized in Table 3. Subjects having an increased number of lacunar lesions were older, more hypertensive, and had stiffer arteries as assessed by baPWV and carotid IMT. Postprandial changes in SBP were also linearly increased with the number of lacunar lesions (Figure 2). Because PHYPO subjects had notable clinical features, i.e. older age (PHYPO 68.7 \pm 7.9 , control 64.8 \pm 9.2 , PHT 65.0 \pm 9.0 years, $\mathrm{p}<0.001)$, higher preprandial SBP $(144 \pm 17,125 \pm 18$, $123 \pm 17 \mathrm{mmHg}, \mathrm{p}<0.001)$ despite a low frequency of antihypertensive medication use (25.4, 26.0, 36.8\%, p=0.034), and faster baPWV $(1668 \pm 323,1562 \pm 324,1628 \pm 303 \mathrm{~cm} / \mathrm{sec}$, $\mathrm{p}=0.001$ ), we performed multivariate analysis to identify factors independently associated with the number of lacunar infarctions. Since there was a step-wise relationship between the number of lacunar infarctions and $\triangle \mathrm{SBP}$ (Figure 2), we used a Poisson regression model, with the number of lacunar infarctions as the dependent variable. Results showed that $\triangle \mathrm{SBP}$ was an independent determinant for the number of lacunar infarctions after adjustment of these covariates (Table 4, model 1 and 2). Although changes in postprandial SBP were not associated with other cerebrovascular diseases, i.e. number of microbleeds or PVH grade (Table 2), we further considered the interrelationship of these asymptomatic cerebrovascular diseases due to their strong positive association with the number of lacunar lesions (Table 3). Multivariate analysis showed that the association of $\triangle$ SBP and $\Delta c$ SBP with lacunar infarction was independent of the existence of PVH and microbleeds (Table 4, models 3 and 4). PHYPO was also significantly associated with the number of lacunar infarctions on analysis adjusted 
for the factors listed in Table 4 (model 3) $(\mathrm{p}=0.047)$. However, no significant association was found in DBP (adjusted for factors in model $1 \mathrm{p}=0.153$ ).

\section{DISCUSSION}

In this cross-sectional study, we found that PHYPO was an independent determinant of asymptomatic lacunar infarction after adjustment for possible covariates. Further, postprandial BP decline was linearly increased with the number of lacunar infarctions. These relationships were also found in an analysis based on central BP, whereas no superiority was seen in the analysis based on central BP. To our knowledge, this is the first study to report a significant association of postprandial BP drop with asymptomatic lacunar infarction in a general population sample with adjustment for known risk factors.

Interestingly, a longitudinal study based on 499 nursing home residents suggested that PHYPO was significantly associated with incident stroke during a 29-month follow-up period [6]. In the present study, we showed that PHYPO was an independent determinant of lacunar infarction, and that lacunar infarction is a potent risk factor for a symptomatic stroke [22]. Silent cerebral infarction might therefore be one factor underlying the suggested relationship between PHYPO and stroke. Although longitudinal data regarding the prognostic significance of PHYPO is currently available only for the frail elderly, our results provide the possibility that PHYPO might be predictive of future stroke not only in frail elderly persons but also in community-dwelling people. Clarification of this issue awaits longitudinal studies based on a general population sample.

Mechanisms to explain the greater postprandial BP decline in subjects with lacunar infarction are unclear. We observed a strong correlation between pre-prandial BP level and the 
degree of postprandial BP decline. Since hypertension is a potent risk factor for lacunar infarction [22], it has been speculated that the interrelationship might be explained by a shared causal factor, i.e. high BP. However, our regression analysis clearly denied a possible interaction of pre-prandial BP level in the relationship between PHYPO and lacunar infarction. A second possible factor for the interrelationship is atherosclerosis. Atherosclerosis increases a risk of lacunar infarction [22] and may also attenuate the baroreceptor reflex, derived from a stretch receptor that acts to compensate for a BP decline [23]. In the present study, we measured carotid IMT, a marker for atherogenic vascular change, and baPWV, which reflects stiffening of arterial walls. Although both arterial parameters were increased with the number of lacunar infarctions, results of the regression analysis indicated that the association between PHYPO and lacunar infarction was independent of these arterial parameters, as well as insulin resistance as evaluated by homeostasis model assessment index for insulin resistance (HOMA-IR). We previously reported that postprandial hypertension but not hypotension was associated with atherosclerosis [24]. Given these findings, the greater drop in postprandial BP in subjects with lacunar infarction might not be simply an epiphenomenon due to the sharing several common pathological factors.

Conversely, a greater postprandial BP decline might be a consequence of the presence of lacunar infarction. Kario et al reported that elderly hypertensive subjects with lacunar infarction showed a greater orthostatic increase or decrease in BP [1 25]. In addition to this short-term BP change, circadian BP pattern, in particular the nighttime BP dipping pattern, has also been suggested to be associated with lacunar infarction in hospitalized patients [26, 27] as well as in a general population sample [28]. Increases in BP variation in various conditions may be a manifestation may reflect the presence of subclinical cerebral damage. 
No significant correlation was observed between postprandial BP drop and other types of cerebral damage, including PVH and MB. We previously reported that postprandial BP decline evaluated by ambulatory BP was associated with not only lacunar infarction but also PVH in elderly patients with essential hypertension [9]. In contrast to our previous study patients, however, the present subjects were community residents with a lower frequency and milder severity of PVH. Since hypertension is a strong risk factor for PVH, and higher preprandial BP was an independent determinant of a postprandial BP decline, the relationship between postprandial BP decline and PVH might be accordingly heightened in patients with essential hypertension.

Several limitations of our study warrant mention. First, we did not serve the same lunch for all subjects, although total calorie was controlled to approximately $600 \mathrm{kcal}$. Carbohydrate-rich meals are reported to predispose patients to more immediate decreases in BP than meals containing mostly protein or fat [29]. Higher sodium and lower potassium intake is an established risk factor for hypertension and might also influence postprandial BP measurement. Further, we did not restrict taking tea or coffee after the lunch because our research was an observational design. However, the dietary constituent of the lunch and caffeine intake would be independent of cerebral disease, as well as a decline in postprandial BP, and non-differential. Second, because our study subjects were participants of a medical check-up program, we did not measure postprandial BP change at different mealtimes. A previous study reported that postprandial BP was greater after breakfast than after lunch or dinner [30]. The association between lacunar infarction and postprandial BP decline might be better manifested by measuring BP change after breakfast. Third, we calculated postprandial BP change from single measurements of pre- and post-prandial BP. The potential effect of 
"regression towards the mean” might not fully excluded. Fourth, we did not have information on atrial fibrillation, a strong risk factor for embolic cerebral infarction. However, because we focused on asymptomatic lacunar infarction and excluded persons with a history of apparent cerebral infarction, this limitation likely had little or no impact on the results.

In summary, excessive postprandial BP decline was associated with asymptomatic lacunar infarction independently of known risk markers. In addition to well-investigated phenomena in BP variability such as postural change, circadian variation, and visit-to-visit variability, greater research effort should be focused on postprandial BP decline as a potential marker for cardiovascular frailty. Further longitudinal studies are needed to clarify the prognostic significance of postprandial BP decline for cerebrovascular disease. 


\section{REFERENCES}

1. Kario K, Eguchi K, Hoshide S, Hoshide Y, Umeda Y, Mitsuhashi T, et al. U-curve relationship between orthostatic blood pressure change and silent cerebrovascular disease in elderly hypertensives. J Am Coll Cardiol. 2002;40:133-141.

2. Verwoert GC, Mattace-Raso FU, Hofman A, Heeringa J, Stricker BH, Breteler MM, et al. Orthostatic hypotension and risk of cardiovascular disease in elderly people: the Rotterdam study. J Am Geriatr Soc. 2008;56:1816-1820

3. Fagard RH, De Cort P. Orthostatic hypotension is a more robust predictor of cardiovascular events than nighttime reverse dipping in elderly. Hypertension. 2010;56:56-61.

4. Rose KM, Eigenbrodt ML, Biga RL, Couper DJ, Light KC, Sharrett AR, et al. Orthostatic hypotension predicts mortality in middle-aged adults: the Atherosclerosis Risk In Communities (ARIC) Study. Circulation. 2006;114:630-636.

5. Masaki KH, Schatz IJ, Burchfiel CM, Sharp DS, Chiu D, Foley D, et al. Orthostatic hypotension predicts mortality in elderly men: the Honolulu Heart Program. Circulation. 1998;98:2290-2295.

6. Aronow WS, Ahn C. Association of postprandial hypotension with incidence of falls, syncope, coronary events, stroke, and total mortality at 29-month follow-up in 499 older nursing home residents. J Am Geriatr Soc. 1997;45:1051-1053.

7. Le Couteur DG, Fisher AA, Davis MW, McLean AJ. Postprandial systolic blood pressure responses of older people in residential care: association with risk of falling.

Gerontology. 2003;49:260-264.

8. Fisher AA, Davis MW, Srikusalanukul W, Budge MM. Postprandial hypotension predicts 
all-cause mortality in older, low-level care residents. J Am Geriatr Soc. 2005;53:13131320.

9. Kohara K, Jiang Y, Igase M, Takata Y, Fukuoka T, Okura T, et al. Postprandial hypotension is associated with asymptomatic cerebrovascular damage in essential hypertensive patients. Hypertension. 1999;33:565-568.

10. Luciano GL, Brennan MJ, Rothberg MB. Postprandial hypotension. Am J Med. 2010;123:281.e1-281.e6.

11. Williams B, Lacy PS, Thom SM, Cruickshank K, Stanton A, Collier D, et al. Differential impact of blood pressure-lowering drugs on central aortic pressure and clinical outcomes: principal results of the Conduit Artery Function Evaluation (CAFE) study. Circulation. 2006;113:1213-1225.

12. Kohara K, Ochi M, Tabara Y, Nagai T, Igase M, Miki T. Arterial stiffness in sarcopenic visceral obesity in the elderly: J-SHIPP study. Int J Cardiol. 2012;158:146-148.

13. Kido T, Tabara Y, Igase M, Ochi N, Uetani E, Nagai T, et al. Postural instability is associated with brain atrophy and cognitive impairment in the elderly: the J-SHIPP study. Dement Geriatr Cogn Disord. 2010;29:379-387.

14. Ochi N, Kohara K, Tabara Y, Nagai T, Kido T, Uetani E, et al. Association of central systolic blood pressure with intracerebral small vessel disease in Japanese. Am $\mathbf{J}$ Hypertens. 2010;23:889-894.

15. Tabara Y, Igase M, Kido T, Ochi N, Miki T, Kohara K. Composition of lower extremity in relation to a high ankle-brachial index. J Hypertens. 2009;27:167-173.

16. Takazawa K, Kobayashi H, Shindo N, Tanaka N, Yamashina A. Relationship between radial and central arterial pulse wave and evaluation of central aortic pressure using the 
radial arterial pulse wave. Hypertens Res. 2007;30:219-228.

17. Takazawa K, Kobayashi H, Kojima I, Aizawa A, Kinoh M, Sugo Y, et al. Estimation of central aortic systolic pressure using late systolic inflection of radial artery pulse and its application to vasodilator therapy. J Hypertens. 2012;30:908-916.

18. Pauca AL, Kon ND, O'Rourke MF. The second peak of the radial artery pressure wave represents aortic systolic pressure in hypertensive and elderly patients. $\mathbf{B r} \mathbf{J}$ Anaesth. 2004;92:651-657.

19. Kohara K, Tabara Y, Tomita H, Nagai T, Igase M, Miki T. Clinical usefulness of the second peak of radial systolic blood pressure for estimation of aortic systolic blood pressure. J Hum Hypertens. 2009;23:538-545.

20. Rosset A, Spadola L, Ratib O. OsiriX: an open-source software for navigating in multidimensional DICOM images. J Digit Imaging. 2004;17:205-216.

21. Yamashina A, Tomiyama H, Takeda K, Tsuda H, Arai T, Hirose K, et al. Validity, reproducibility, and clinical significance of noninvasive brachial-ankle pulse wave velocity measurement. Hypertens Res. 2002;25:359-364.

22. Vermeer SE, Longstreth WT Jr, Koudstaal PJ. Silent brain infarcts: a systematic review. Lancet Neurol. 2007;6:611-619.

23. Gianaros PJ, Jennings JR, Olafsson GB, Steptoe A, Sutton-Tyrrell K, Muldoon MF, et al. Greater intima-media thickness in the carotid bulb is associated with reduced baroreflex sensitivity. Am J Hypertens. 2002;15:486-491.

24. Uetani E, Tabara Y, Igase M, Guo H, Kido T, Ochi N, et al. Postprandial hypertension, an overlooked risk marker for arteriosclerosis. Atherosclerosis. 2012;224:500-505.

25. Eguchi K, Kario K, Hoshide S, Hoshide Y, Ishikawa J, Morinari M, et al. Greater change 
of orthostatic blood pressure is related to silent cerebral infarct and cardiac overload in hypertensive subjects. Hypertens Res. 2004;27:235-41.

26. Kukla C, Sander D, Schwarze J, Wittich I, Klingelhöfer J. Changes of circadian blood pressure patterns are associated with the occurence of lucunar infarction. Arch Neurol. 1998;55:683-688.

27. Yamamoto Y, Akiguchi I, Oiwa K, Hayashi M, Ohara T, Ozasa K. The relationship between 24-hour blood pressure readings, subcortical ischemic lesions and vascular dementia. Cerebrovasc Dis. 2005;19:302-308.

28. Watanabe N, Imai Y, Nagai K, Tsuji I, Satoh H, Sakuma M, et al. Nocturnal blood pressure and silent cerebrovascular lesions in elderly Japanese. Stroke. 1996;27:13191327.

29. Gentilcore D, Hausken T, Meyer JH, Chapman IM, Horowitz M, Jones KL. Effects of intraduodenal glucose, fat, and protein on blood pressure, heart rate, and splanchnic blood flow in healthy older subjects. Am J Clin Nutr. 2008;87:156-161.

30. Puisieux F, Bulckaen H, Fauchais AL, Drumez S, Salomez-Granier F, Dewailly P. Ambulatory blood pressure monitoring and postprandial hypotension in elderly persons with falls or syncopes. J Gerontol A Biol Sci Med Sci. 2000;55:M535-M540. 


\section{FIGURE LEGEND}

\section{Figure 1 Correlation between postprandial changes in SBP and cSBP}

Postprandial changes in blood pressure were calculated from two readings, one obtained just before lunch and the second 30 min after lunch. Absolute pressure of the late systolic peak was obtained by calibrating the radial waveform with brachial SBP and considered to be the cSBP.

Figure 2 Postprandial changes in SBP and cSBP by the number of lacunar infarctions

Values are mean \pm standard deviation. Numbers of subjects in each subgroup are represented in the column. Statistical differences were assessed by analysis of variance. 
Table 1 Clinical characteristics of study subjects $(n=1,308)$

\begin{tabular}{|c|c|c|}
\hline Age (years) & \multicolumn{2}{|c|}{$65.2 \pm 9.1$} \\
\hline Sex (male/female) & \multicolumn{2}{|c|}{$514 / 794$} \\
\hline $\operatorname{BMI}\left(\mathrm{kg} / \mathrm{m}^{2}\right) \mathrm{s}$ & \multicolumn{2}{|c|}{$23.3 \pm 3.1$} \\
\hline Blood pressure & Preprandial & Postprandial \\
\hline SBP (mmHg) & $126 \pm 18$ & $123 \pm 18$ \\
\hline cSBP (mmHg) & $119 \pm 19$ & $111 \pm 18$ \\
\hline DBP (mmHg) & $72 \pm 11$ & $67 \pm 11$ \\
\hline AIx (\%) & $89 \pm 12$ & $81 \pm 13$ \\
\hline AIx at HR75 (\%) & $85 \pm 11$ & $79 \pm 12$ \\
\hline HR (betas/min.) & $66 \pm 10$ & $71 \pm 10$ \\
\hline Antihypertensive medication (\%) & \multicolumn{2}{|c|}{27.0} \\
\hline Beta-blockers (\%) & \multicolumn{2}{|c|}{3.2} \\
\hline Alpha-blockers (\%) & \multicolumn{2}{|c|}{0.8} \\
\hline Calcium-channel blockers (\%) & \multicolumn{2}{|c|}{17.3} \\
\hline Diuretics (\%) & \multicolumn{2}{|c|}{1.5} \\
\hline Angiotensin-converting enzyme inhibitors (\%) & \multicolumn{2}{|c|}{1.2} \\
\hline Angiotensin II receptor blockers (\%) & \multicolumn{2}{|c|}{13.0} \\
\hline \multicolumn{3}{|l|}{ Arterial parameters } \\
\hline baPWV (cm/sec) & \multicolumn{2}{|c|}{$1578 \pm 323$} \\
\hline Carotid IMT (mm) & \multicolumn{2}{|c|}{$0.79 \pm 0.15$} \\
\hline \multicolumn{3}{|l|}{ Plasma markers } \\
\hline Total cholesterol (mg/dl) & \multicolumn{2}{|c|}{$219 \pm 37$} \\
\hline HDL cholesterol (mg/dl) & \multicolumn{2}{|c|}{$67 \pm 18$} \\
\hline Glucose (mg/dl) & \multicolumn{2}{|c|}{$103 \pm 17$} \\
\hline Insulin $(\mu \mathrm{U} / \mathrm{ml})$ & \multicolumn{2}{|c|}{$5.80 \pm 3.83$} \\
\hline HOMA-IR & \multicolumn{2}{|c|}{$1.51 \pm 1.15$} \\
\hline $\mathrm{BNP}(\mathrm{pg} / \mathrm{ml})$ & \multicolumn{2}{|c|}{$31.3 \pm 30.1$} \\
\hline \multicolumn{3}{|l|}{ Silent cerebrovascular disease } \\
\hline Number of lacunar infarction $(0 / 1 / 2 / 3 / 4)$ & \multicolumn{2}{|c|}{$1,200 / 82 / 18 / 7 / 1$} \\
\hline PVH grade $(0 / 1 / 2 / 3 / 4)$ & \multicolumn{2}{|c|}{$602 / 556 / 132 / 14 / 4$} \\
\hline Number of microbleeds $(0 / 1 / 2 / 3<)$ & \multicolumn{2}{|c|}{$1,234 / 55 / 11 / 4$} \\
\hline
\end{tabular}

Homeostasis model assessment index for insulin resistance (HOMA-IR) was calculated as insulin $(\mu \mathrm{U} / \mathrm{ml}) \times$ glucose $(\mathrm{mg} / \mathrm{dl}) / 405$. Intima media thickness (IMT) at the common carotid artery was measured by ultrasonography. Brachial-to-ankle pulse wave velocity (baPWV) was calculated from the time interval between the wave fronts of the brachial and ankle waveforms, and the path length from the brachial to ankle. BMI, body mass index; SBP, systolic blood pressure; cSBP, central aortic SBP; DBP, diastolic blood pressure; AIx, augmentation index; HR, heart rate; BNP, B-type natriuretic peptide; $\mathrm{PVH}$, periventricular hyperintensity. 
Table 2 Frequency of silent cerebrovascular diseases by postprandial BP change

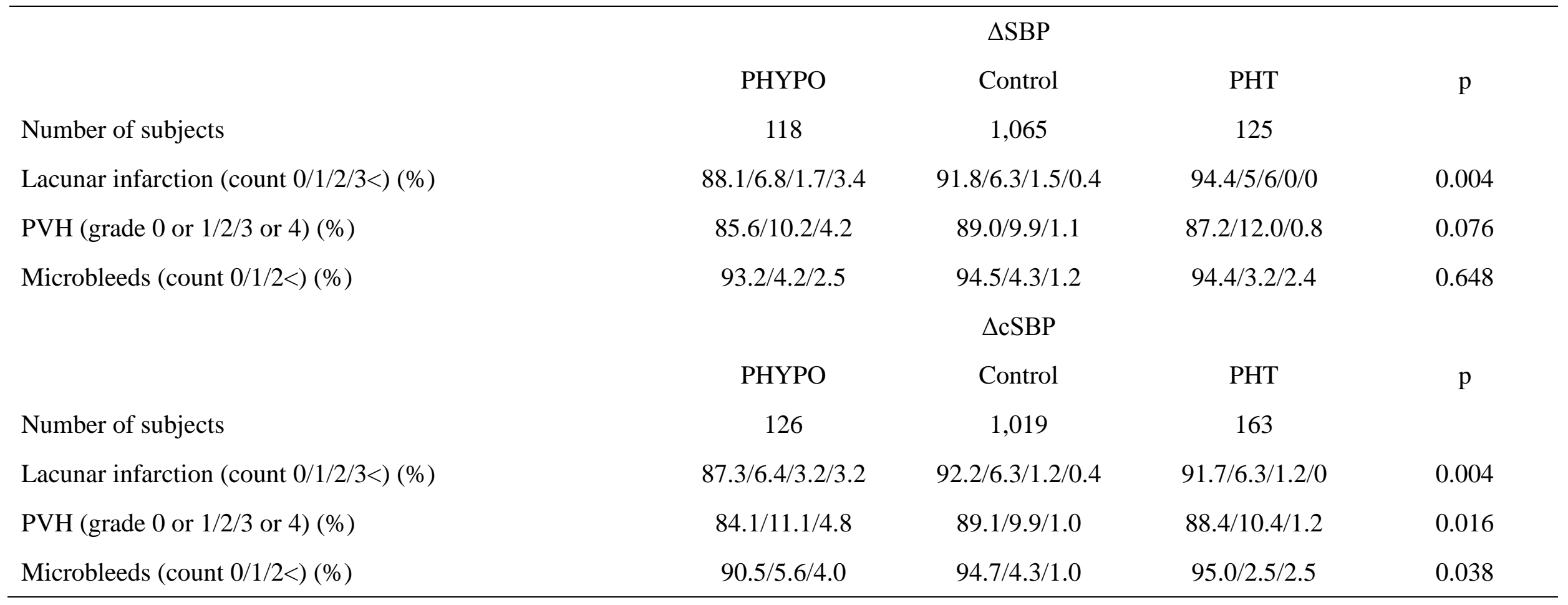

Subjects were classified into three groups according to postprandial change in SBP $(\Delta \mathrm{SBP})$ [postprandial hypotension (PHYPO: $\triangle \mathrm{SBP} \leq-20$ $\mathrm{mmHg}$ ), Control $(-10<\Delta \mathrm{SBP} \leq 10 \mathrm{mmHg}$ ), and postprandial hypertension (PHT: $\Delta \mathrm{SBP}>10 \mathrm{mmHg})]$, or postprandial change in cSBP $(\Delta \mathrm{cSBP})$ [PHYPO $\Delta \mathrm{cSBP} \leq-25 \mathrm{mmHg}$, Control $-25<\Delta \mathrm{cSBP} \leq 5 \mathrm{mmHg}$, and PHT $\Delta \mathrm{cSBP}>5 \mathrm{mmHg}$ ). PVH was classified into five grades, namely grade 0 , absent or only a "rim”; grade 1, limited lesion-like “caps”; grade 2, irregular "halo”; grade 3, irregular margins and extension into the deep white matter; and grade 4, extension into the deep white matter and subcortical portion. Frequency difference was assessed by chi-squared test. 
Table 3 Differences in clinical parameters by the number of lacunar infarctions

\begin{tabular}{|c|c|c|c|c|c|}
\hline & \multicolumn{4}{|c|}{ Number of lacunar infarction } & \multirow[b]{2}{*}{$\mathrm{p}$} \\
\hline & $\begin{array}{c}0 \\
(1,200)\end{array}$ & $\begin{array}{c}1 \\
(82)\end{array}$ & $\begin{array}{c}2 \\
(18)\end{array}$ & $\begin{array}{l}3< \\
(8)\end{array}$ & \\
\hline Age (years) & $64.8 \pm 9.2$ & $69.9 \pm 7.2$ & $69.1 \pm 5.9$ & $69.4 \pm 6.9$ & $<0.001$ \\
\hline Sex (male\%) & 39.1 & 42.7 & 38.9 & 37.5 & 0.934 \\
\hline $\mathrm{BMI}\left(\mathrm{kg} / \mathrm{m}^{2}\right)$ & $23.2 \pm 3.1$ & $23.9 \pm 3.2$ & $23.1 \pm 2.6$ & $23.3 \pm 4.1$ & 0.321 \\
\hline Preprandial SBP (mmHg) & $125 \pm 18$ & $135 \pm 19$ & $137 \pm 23$ & $128 \pm 17$ & $<0.001$ \\
\hline Antihypertensive medication (\%) & 24.7 & 53.7 & 61.1 & 25.0 & $<0.001$ \\
\hline baPWV (cm/sec) & $1560 \pm 316$ & $1786 \pm 342$ & $1704 \pm 326$ & $1797 \pm 337$ & $<0.001$ \\
\hline Carotid IMT (mm) & $0.78 \pm 0.15$ & $0.84 \pm 0.14$ & $0.81 \pm 011$ & $0.85 \pm 0.13$ & 0.005 \\
\hline HDL cholesterol (mg/dl) & $67 \pm 18$ & $65 \pm 19$ & $68 \pm 19$ & $70 \pm 19$ & 0.769 \\
\hline $\mathrm{BNP}(\mathrm{pg} / \mathrm{ml})$ & $30.5 \pm 29.4$ & $39.1 \pm 34.7$ & $42.5 \pm 38.4$ & $49.8 \pm 35.1$ & $0.031^{\#}$ \\
\hline HOMA-IR & $1.49 \pm 1.14$ & $1.83 \pm 1.30$ & $1.43 \pm 0.99$ & $1.33 \pm 0.77$ & $0.037^{\#}$ \\
\hline PVH (grade 0 or $1 / 2 / 3$ or 4 ) (\%) & $90.8 / 8.3 / 0.9$ & $67.1 / 25.6 / 7.3$ & $50.0 / 50.0 / 0$ & $62.5 / 25.0 / 12.5$ & $<0.001$ \\
\hline Microbleeds (count $0 / 1 / 2<)(\%)$ & $95.8 / 3.3 / 0.8$ & 81.7/13.4/4.9 & $66.7 / 16.8 / 16.8$ & $62.5 / 12.5 / 25.0$ & $<0.001$ \\
\hline
\end{tabular}

Differences in numeric variables were assessed by an analysis of variance while frequency differences were evaluated by a chi-squared test. \#, statistical analysis was performed using a log-normalized value. 
Table 4 Multiple Poisson regression analysis for the number of lacunar infarctions

\begin{tabular}{|c|c|c|c|c|c|c|c|c|}
\hline & \multicolumn{2}{|c|}{ Model 1} & \multicolumn{2}{|c|}{ Model 2} & \multicolumn{2}{|c|}{ Model 3} & \multicolumn{2}{|c|}{ Model 4} \\
\hline & coefficient & $\mathrm{p}$ & coefficient & $\mathrm{p}$ & coefficient & $\mathrm{p}$ & coefficient & $\mathrm{p}$ \\
\hline Age (years) & 0.052 & $<0.001$ & 0.036 & 0.006 & 0.014 & 0.283 & 0.016 & 0.212 \\
\hline BMI $\left(\mathrm{kg} / \mathrm{m}^{2}\right)$ & 0.013 & 0.662 & 0.032 & 0.326 & 0.028 & 0.388 & 0.024 & 0.470 \\
\hline Current smoking & 0.963 & 0.001 & 0.993 & $<0.001$ & 1.017 & $<0.001$ & 1.007 & $<0.001$ \\
\hline Preprandial SBP (mmHg) & 0.009 & 0.075 & -0.001 & 0.895 & 0.002 & 0.761 & 0.004 & 0.471 \\
\hline Postprandial changes in SBP (mmHg) & -0.019 & 0.014 & -0.026 & 0.001 & -0.022 & 0.004 & & \\
\hline Postprandial changes in cSBP (mmHg) & & & & & & & -0.014 & 0.048 \\
\hline Antihypertensive medication & 0.783 & $<0.001$ & 0.739 & $<0.001$ & 0.499 & 0.007 & 0.490 & 0.008 \\
\hline PVH (grade 2 or higher) & & & & & 1.007 & $<0.001$ & 1.016 & $<0.001$ \\
\hline Microbleeds (more than one lesion) & & & & & 0.995 & $<0.001$ & 1.011 & $<0.001$ \\
\hline
\end{tabular}




\section{FIGURE 1}

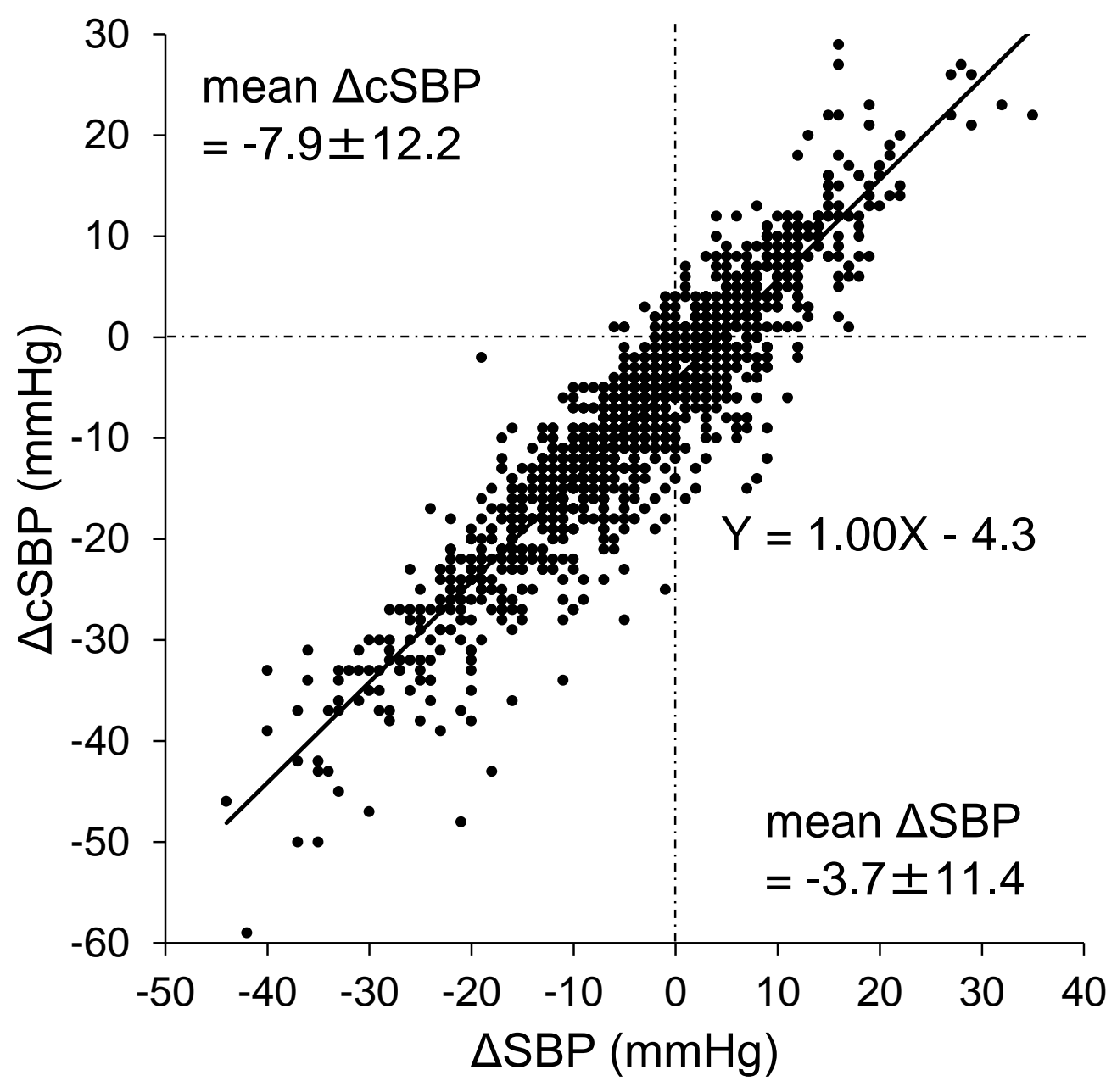




\section{FIGURE 2}

A Number of lacunar infarction

B Number of lacunar infarction
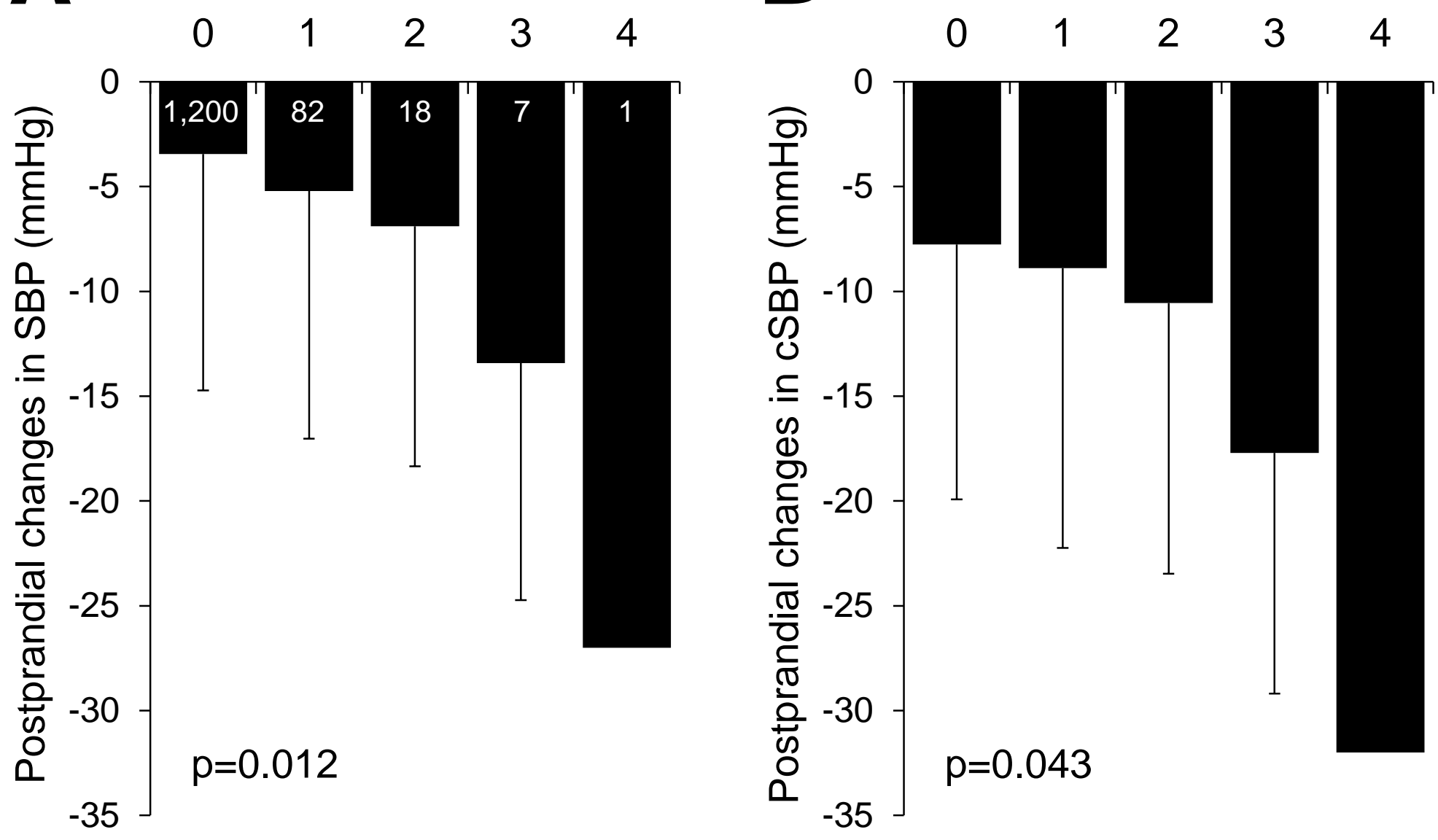